\title{
Visualization of Sialic Acid Produced on Bacterial Cell Surfaces by Lectin Staining
}

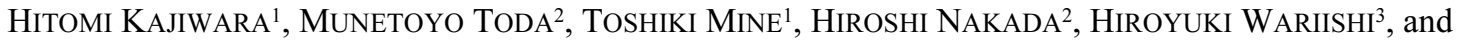 \\ TAKESHI YAMAMOTO ${ }^{1 *}$ \\ ${ }^{1}$ Glycotechnology Business Unit, Japan Tobacco Inc., 700 Higashibara, Iwata, Shizuoka 438-0802, Japan; \\ ${ }^{2}$ Department of Biotechnology, Faculty of Engineering, Kyoto Sangyo University, Motoyama, Kamigamo, Kita-ku, \\ Kyoto, Kyoto 603-8555, Japan; and ${ }^{3}$ Department of Forest and Forest Products Sciences, Graduate School of \\ Bioresource and Bioenvironmental Sciences, Kyusyu University, 6-10-1, Hakozaki, Higashi-ku, Fukuoka, \\ Fukuoka 812-8581, Japan
}

(Received March 12, 2010_Accepted April 16, 2010_-Published online May 13, 2010)

Oligosaccharides containing $N$-acetylneuraminic acid on the cell surface of some pathogenic bacteria are important for host-microbe interactions. $N$-acetylneuraminic acid (Neu5Ac) plays a major role in the pathogenicity of bacterial pathogens. For example, cell surface sialyloligosaccharide moieties of the human pathogen Haemophilus influenzae are involved in virulence and adhesion to host cells. In this study, we have established a method of visualizing Neu5Ac linked to a glycoconjugate on the bacterial cell surface based on lectin staining. Photobacterium damselae strain JT0160, known to produce $\alpha$-2,6-sialyltransferase, was revealed to possess Neu5Ac by HPLC. Using the strain, a strong Sambucus sieboldiana lectin-binding signal was detected. The bacteria producing $\alpha-2,6$-sialyltransferases could be divided into two groups: those with a lot of $\alpha-2,6$-linked Neu5Ac on the cell surface and those with a little. In the present study, we developed a useful method for evaluating the relationship between Neu5Ac expression on the cell surface and the degree of virulence of marine bacteria.

Key words: $N$-acetylneuraminic acid, lectin staining, Photobacterium, sialyloligosaccharide, Vibrio

\section{Introduction}

Sialic acids are important components of carbohydrate chains commonly occurring at the terminal position to form glycoconjugates, including glycoproteins and glycolipids (1, 16). $\mathrm{N}$-acetylneuraminic acid (Neu5Ac) is a major sialic acid linked to glycoconjugates, and the sialyloligosaccharides of glycoconjugates play important roles in many biological processes including inflammatory and immunological responses, cell-cell recognition, cancer metastasis and viral infection $(5,15)$. Among the glycoconjugates, lipooligosaccharides, lipopolysaccharides and glycoproteins are located on the surface of bacteria pathogenic to humans such as Campylobacter jejuni and Haemophilus influenzae, and their sugar chain structures have been determined $(2,3,7,20)$. C. jejuni and $H$. influenzae are associated with Guillain-Barré syndrome and Fisher syndrome, respectively. By mimicking the carbohydrates of human host cells, the bacterial pathogen can evade the host immune systems, which are crucial for self/non-self recognition. The terminal oligosaccharide moieties of both $C$. jejuni and $H$. influenzae are identical to those of gangliosides, the general term for compounds composed of a glycosphingolipid with one or more sialic acid linked to the sugar chains, such as GM1 (Gal- $\beta-1,3-$ GalNAc$\beta-1,4-[N e u 5 A c-\alpha-2,3-]$ Gal- $\beta-1,4-G l c-\beta-1,1$-ceramide), GD3 (Neu5Ac- $\alpha-2,8-N e u 5 A c-\alpha-2,3-G a l-\beta-1,4-G l c-\beta-1,1-c e r a m i d e)$, GT1a(Neu5Ac- $\alpha-2,8-N e u 5 A c-\alpha-2,3-G a l-\beta-1,3-G a l N A c-\beta-1,4-$ [Neu5Ac- $\alpha-2,3-]$ Gal- $\beta-1,4-G l c-\beta-1,1-c e r a m i d e)$, GQ1b (Neu5Ac-

\footnotetext{
* Corresponding author. E-mail: takeshi.yamamoto@jt.com; Tel: +81-538-32-7389; Fax: +81-538-32-6046.
}

$\alpha-2,8-N e u 5$ Ac- $\alpha-2,3-$ Gal- $\beta-1,3-$ GalNAc- $\beta-1,4-[$ Neu5Ac- $\alpha-$ $2,8$-Neu 5 Ac- $\alpha-2,3-]$ Gal- $\beta$ - $1,4-G l c-\beta-1,1$-ceramide) and so on $(3,7)$, and these oligosaccharide moieties are involved in virulence and adhesion to host cells (20). These cases are thought to be just the tip of the iceberg when it comes to the biological functions including host-microbe interactions in which sialyloligosaccharides (sialosides) participate. Except for bacteria pathogenic to humans, there are few examples where the function of the bacterial carbohydrate chain has been clarified.

As the sialyloligosaccharides of glycoconjugates play important roles in many biological processes, an abundant supply of sialosides is essential for a detailed investigation of the biological functions of sialosides. To produce these sialosides enzymatically, we have been screening bacteria for sialyltransferase activities and isolated over 20 that produce sialyltransferases (18). Many of these bacteria are classified into the genera Photobacterium and Vibrio (18). Although we have clarified the enzymatic characteristics of the sialyltransferases, we have not obtained structural information on the oligosaccharides produced by these bacteria. To obtain information about the biological roles of oligosaccharides produced by bacteria, many bacteria having unique oligosaccharides are needed. In this regards, marine bacteria producing sialyltransferases are thought to be good candidates.

Among sialytransferase-producers, Photobacterium damselae is known as a causative agent of wound infection, ulceration, and pasteurellosis $(4,6,8-10)$. Not only $P$. damselae subsp. piscicida but also $P$. damselae subsp. damselae contained sialic acid as part of either a poly- 
saccharide molecule or a glycoprotein molecule (6). So, it is clear that the species possess unique systems producing sialoside. Consequently, fish or human pathogens from marine environments are worth testing for unique sialosides, as sialyltransferase producers. However, the determination of sialyltransferase activity or sialosides of bacteria is time consuming. Thus, it is important to develop a novel tool for detecting the sialic acid expressed on bacterial surfaces. As sialic acids are key molecules for host-microbe interactions, their direct visualization provides great advantages for analyzing host-microbe interactions, especially assessing the virulence of bacteria. In this study, we report on a method for visualizing the Neu5Ac residues on bacterial cell surfaces based on lectin staining.

\section{Materials and Methods}

\section{Chemicals and materials}

The Alexa 594-labeled streptavidin solution and Prolong Gold antifade reagent were purchased from Invitrogen (Carlsbad, CA, USA); Cytidine-5'-monophospho- $N$-acetylneuraminic acid (CMPNeu5Ac) from Japan Food and Liquor Alliance (Kyoto, Japan); CMP-[4,5,6,7,8,9- $\left.{ }^{14} \mathrm{C}\right]-\mathrm{Neu} 5 \mathrm{Ac}$ from Amersham Biosciences (Little Chalfont,UK);1,2-diamino-4,5-methylenedioxybenzene(DMB)from Takara Bio (Otsu, Japan); MAS-coated glass slides (glass slides coated with a material preventing cell detachment) from Matsunami Glass (Osaka, Japan); Lactose from Wako Pure Chemical Industries (Osaka, Japan); Luria-Bertani (LB) broth, Type Miller, from BD Bioscience (Sparks, MD, USA); Nutrient broth from BectonDickinson (Franklin Lakes, NJ, USA); Sialidase (Arthrobactor ureafaciens origin) from Sigma (St Louis, MO, USA); Sambucus sieboldiana lectin (SSA) from Seikagaku Kogyo (Tokyo, Japan); and STR ODR-II column from Shinwa Chemical Industries (Kyoto, Japan). All other reagents were of a commercially available analytical grade.

\section{Bacteria}

Photobacterium sp. JT-ISH-224 was isolated from the gut of a Japanese barracuda, Sphyraena pinguis (14); Photobacterium phosphoreum JT-ISH-467 from the outer skin of Japanese common squid, Todarodes pacificus (13); P. damselae JT0160 from seawater (17); and Photobacterium leiognathi JT-SHIZ-145 from the outer skin of Japanese squid, Loliolus japonica (19). P. damselae strains ATCC 33536, ATCC 33537, ATCC 33538, ATCC 33539 and ATCC35083 were purchased from the American Type Culture Collection (Manassas, VA, USA).

\section{Mild acid hydrolysis of bacteria}

Mild acid hydrolysis was used to release sialic acids from the surface of bacteria as reported previously (6). In brief, bacterial cells were suspended in $0.1 \mathrm{M} \mathrm{H}_{2} \mathrm{SO}_{4}$, and incubated for $1 \mathrm{~h}$ at $80^{\circ} \mathrm{C}$. The cell suspension was centrifuged, and the supernatant containing sialic acid was used.

\section{Detection and Quantification of Neu5Ac by HPLC}

The sialic acids were labeled with DMB according to the manufacturer's instructions. High performance liquid chromatography (HPLC) was carried out using an STR ODR-II column with conditions as follows: Solvent, acetonitrile/methnol/ $\mathrm{H}_{2} \mathrm{O}=9 / 7 / 84(\mathrm{v} / \mathrm{v} / \mathrm{v})$; fluorescence, Ex. $373 \mathrm{~nm}$ and Em. $448 \mathrm{~nm}$; flow rate, $0.9 \mathrm{~mL} \mathrm{~min}{ }^{-1}$.

\section{Lectin staining}

Lectin staining was carried out as follows: $P$. damselae JT0160, $P$. leiognathi JT-SHIZ-145, $P$. phosphoreum JT-ISH-467 and Photobacterium sp. JT-ISH-224 were cultivated at $25^{\circ} \mathrm{C}$ for $18 \mathrm{~h}$ on a rotary shaker, using nutrient broth supplemented with $2 \%(\mathrm{w} / \mathrm{v})$ $\mathrm{NaCl}$. Escherichia coli strain BL21 was cultivated at $37^{\circ} \mathrm{C}$ for 18 $\mathrm{h}$ on a rotary shaker, using LB broth. The bacterial cells were collected by centrifugation $\left(8,000 \times \mathrm{g}, 15 \mathrm{~min}, 4^{\circ} \mathrm{C}\right)$ and resuspended in $25 \mathrm{mM}$ Tris- $\mathrm{HCl}$ buffer ( $\mathrm{pH}$ 7.5). These suspensions were spotted onto glass slides, fixed with a $4 \%(\mathrm{w} / \mathrm{v})$ paraformaldehyde solution at room temperature for $15 \mathrm{~min}$, and blocked with a $5 \%(\mathrm{w} / \mathrm{v})$ bovine serum albumin (BSA) phosphate-buffered saline (PBS) solution. After the glass slides were washed, biotin-labeled SSA $\left(5 \mu \mathrm{g} \mathrm{mL}^{-1}\right)$ was added at room temperature for $2 \mathrm{~h}$, and the cells were washed four times with PBS. Alexa 594-labeled streptavidin solution $\left(5 \mu \mathrm{g} \mathrm{mL} \mathrm{m}^{-1}\right)$ was added and the incubation continued at room temperature for $1 \mathrm{~h}$. After five washes with PBS, the cells were mounted using Prolong Gold antifade reagent (Invitrogen), and observed by differential interference contrast (DIC) microscopy and fluorescence microscopy. In case of the sialidase-treated $P$. damselae JT0160 cells, the sialyloligosaccharides on the cells were digested with sialidase and stained as described above. The sialidase treatment was carried out at $37^{\circ} \mathrm{C}$ for $1 \mathrm{~h}$.

\section{Results and Discussion}

Sialyloligosaccharides are key molecules for the establishment of not only an infection but also any other type of host-microbe interaction $(4,10)$. To fully understand the biological functions of bacterial sialyloligosaccharides, a series of bacteria producing various types of sialyloligosaccharides and/or sialyltransferases are required. In fact, we have found that some Photobacterium and Vibrio spp. isolated from marine environments produce unique sialyltransferases $(12,13,17,19)$. Using these marine bacteria, we developed a refined method of screening for sialyloligosaccharide producers and/or for sialyltransferases indirectly. The bacteria producing $\alpha-2,6$-sialyltransferases were found to express Neu5Ac on the cell surface. Furthermore, the bacteria tested could be divided into two groups: those with a lot of $\alpha-2,6$-linked Neu5Ac on the cell surface and those with a little. Although the structures of the sialyloligosaccharides produced by these bacteria are still unknown, the results should help reveal the relationship between the expression of $\alpha-2,6$-linked Neu5Ac and the degree of virulence of these bacteria.

\section{Staining by S. sieboldiana lectin (SSA)}

SSA is known to recognize the Neu5Ac- $\alpha-2,6-\mathrm{Gal}$ or Neu5Ac- $\alpha-2,6-$ GalNAc structure of sialyloligosaccharides on glycoconjugates (11). Cells of $P$. damselae JT0160, $P$. leiognathi JT-SHIZ-145, $P$. phosphoreum JT-ISH-467 and Photobacterium sp. JT-ISH-224, were incubated with biotinlabeled SSA, and observed under the DIC and fluorescence microscopes. As shown in Fig. 1, SSA bound to P. damselae JT0160, P. leiognathi JT-SHIZ-145 and Photobacterium sp. JT-ISH-224. As all these Photobacterium strains have $\alpha-2,6-$ sialyltransferases, the staining indirectly detected $\alpha-2,6-$ sialyltransferase-producing bacteria. The results also indicate that Neu5Ac- $\alpha-2,6-$ Gal or Neu5Ac- $\alpha-2,6-$ GalNAc was present on these bacteria. SSA did not bind to $P$. phosphoreum JT-ISH-467, which produces only $\alpha$-2,3-sialyltransferase (Fig. 1). Therefore, the SSA lectin may be useful in a semiqualitative analysis to screen for not only Neu5Ac- $\alpha-2,6-\mathrm{Gal}$ or Neu5Ac- $\alpha-2,6-G a l N A c$ linked-glycoconjugate-producing bacteria but also $\alpha-2,6$-sialyltransferase producers.

No Neu5Ac residues were detected on the surface of either $P$. damselae JT0160 cells treated with sialidase or E. coli 


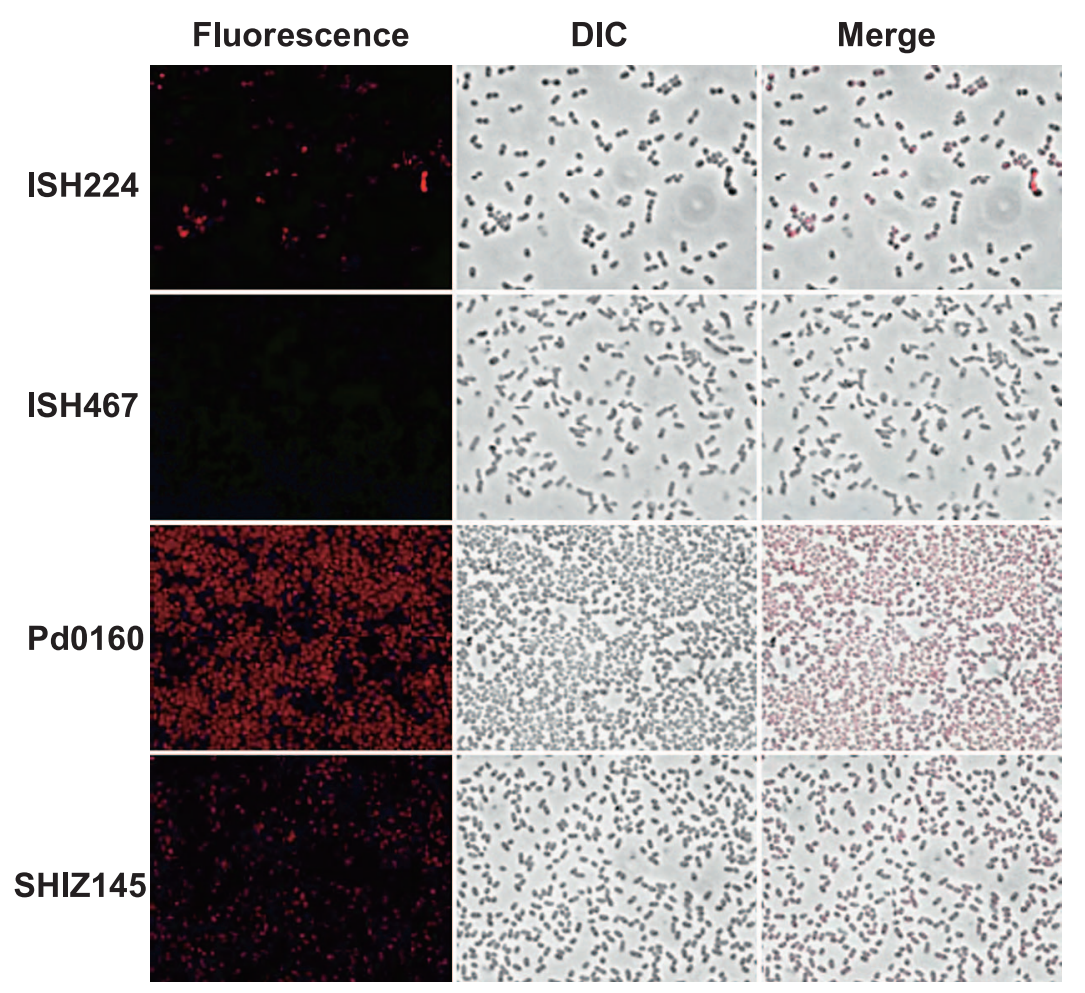

Fig. 1. Detection of Neu5Ac residues on marine bacterial cells using biotin-labeled SSA. The SSA lectin specific for Neu5Ac- $\alpha-2,6-\mathrm{Gal} / \mathrm{GalNAc}$ bound to cell surfaces of marine bacteria producing $\alpha-2,6$-sialyltransferases (red staining in the SSA column, detected with fluorescein Alexa 594labeled streptavidin). ISH224, Photobacterium sp. JT-ISH-224; ISH467, Photobacterium phosphoreum JT-ISH-467; Pd0160, Photobacterium damselae JT0160; SHIZ145, Photobacterium leiognathi JT-SHIZ-145; DIC, results of observation by differential interference contrast microscopy; Fluorescence, results of SSA lectin staining observed using fluorescence microscopy; and Merge, merged results for DIC and Fluorescence.

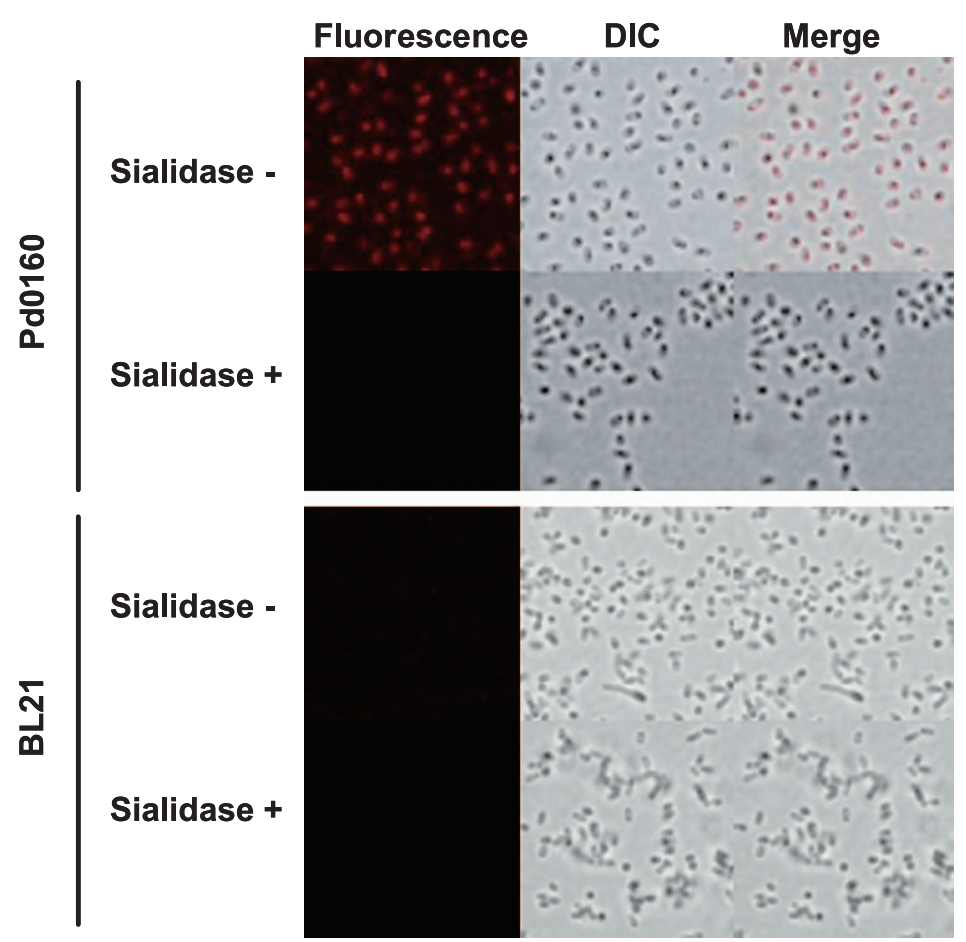

Fig. 2. SSA lectin staining of sialidase-treated Photobacterium damselae JT0160 cells (Pd0160) and Escherichia coli strain BL21 cells (BL21). 


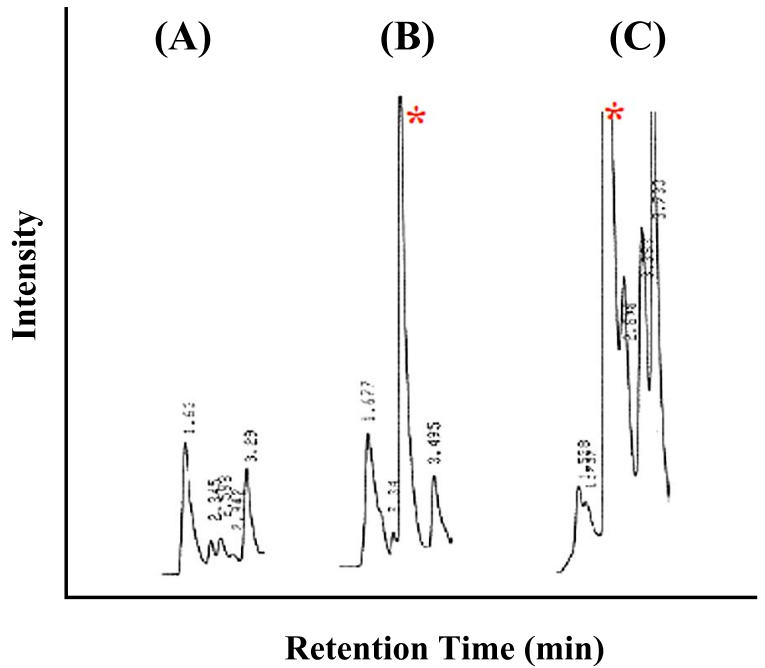

Fig. 3. HPLC of Neu5Ac and the release of Neu5Ac from Photobacterium damselae JT0160 cells with fluorescence reagent (DMB): (A) only DMB, (B) Neu5Ac labeled with DMB, (C) hydrolytic fraction labeled with DMB. *DMB-labeled Neu5Ac.

BL21 cells that did not produce Neu5Ac by staining SSA (Fig. 2). These results strongly indicated that Neu5Ac residues and its linkage on cell surfaces could be detected specifically by the method developed in this study.

\section{Determination of Neu5Ac-linked oligosaccharide production} by P. damselae JT0160

$P$. damselae JT0160 was isolated from seawater (17), and produces $\alpha$-2,6-sialyltransferase (17). As shown in Fig. 1, SSA bound to the $P$. damselae JT0160 cells well compared to the Photobacterium sp. JT-ISH-224 and P. leiognathi JT-SHIZ-145 cells. Then, compounds obtained from mild acid treatment of $P$. damselae JT0160 cells were analyzed by HPLC. As shown in Fig. 3, DMB-labeled sialic acid released from the acid-treated $P$. damselae JT0160 cells was detected (Fig. 3C). By comparing the retention time of Neu5Ac-DMB (Fig. 3B), the sialic acid released from P. damselae JT0160 cells was identified as Neu5Ac (Fig. 3C). The estimated amount of Neu5Ac was 162.5 pmol ( $\mathrm{g}$ wet weight of the cells $)^{-1}$. Previously, three strains of $P$. damselae subsp. damselae and forty strains of $P$. damselae subsp. piscicida were examined for the presence of sialic acid using a commercially available immunoblotting kit (6). Many of the bacteria were found to contain sialic acid as part of either a polysaccharide molecule or a glycoprotein molecule (6). However, identification of the sialic acid residue was not reported. To our knowledge, this is the first report to obtain evidence of the production of Neu5Ac-linked glycoconjugates by $P$. damselae.

Although many sialic acid-expressing bacteria are pathogenic, there is almost no information about the structure of the carbohydrate chains that exist on the cell surface of pathogenic marine bacteria causing diseases in fish. The results of this study should provide valuable information about the surface sialyloligosaccharides of fish pathogens.

\section{Acknowledgements}

The authors thank Professor Sanji Matsushima and Professor
Yoshisuke Nishi, Nagahama Institute of Bio-Science and Technology, for valuable advice and Ms. Asumi Minami and Ms. Sachiyo Higashi for excellent help with the laboratory work. The authors also thank Professor Tomoo Sawabe, Hokkaido University, for critical reading.

\section{References}

1. Angata, T., and A. Varki. 2002. Chemical diversity in the sialic acids and related $\alpha$-keto acids: An evolutionary perspective. Chem. Rev. 102:439-469.

2. Aspinall, G.O., A.G. McDonald, and H. Pang. 1994. Lipopolysaccharides of Campylobacter jejuni serotype O:19: Structures of O-antigen chains from the serostrain and two bacterial isolates from patients with the Guillain-Barré syndrome. Biochemistry 33:250-255.

3. Aspinall, G.O., A.G. McDonald, H. Pang, L.A. Kurjanezyk, and J.L. Penner. 1994. Lipopolysaccharides of Campylobacter jejuni serotype O:19: Structures of core oligosaccharide regions from the serostrain and two bacterial isolates from patients with the Guillain-Barré syndrome. Biochemistry 33:241-249.

4. Austin, B. 2010. Vibrios as causal agents of zoonoses. Vet. Microbiol. 140:310-317.

5. Gagneux, P., and A. Varki. 1999. Evolutionary considerations in relating oligosaccharide diversity to biological function. Glycobiology 9:747-755.

6. Jung, T.S., K.D. Thompson, and A. Adams. 2001. A comparison of sialic acid between different isolates of Photobacterium damselae subsp. piscicida. Fish Pathol. 36:217-224.

7. Koga, M., M. Gilbert, J. Li, S. Koike, M. Takahashi, K. Furukawa, K. Hirata, and N. Yuki. 2005. Antecedent infections in Fisher syndrome: A common pathogenesis of molecular mimicry. Neurology 64:16051611.

8. Love, M., D. Teebken-Fisher, and M. Mecca. 1981. The marine bacterium Vibrio damsela sp. causes skin ulcers on the damselfish Chromis punctipinnis: Association with human infections. Science 214:1139-1140.

9. Matsuoka, S., and S. Kamada. 1995. Discharge of pasteurella piscicida cells from experimentally infected yellowtail. Fish Pathol. 30:221-225.

10. Romalde, J.L. 2002. Photobacterium damselae subsp. piscicida: An integrated view of a bacterial fish pathogen. Int. Microbiol. 5:3-9.

11. Shibuya, N., K. Tazaki, Z. Song, G.E. Tarr, I.J. Goldstein, and W.J.A. Peumans. 1989. Comparative study of bark lectins from three elderberry (Sambucus) species. J. Biochem. 106:1098-1103.

12. Takakura, Y., H. Tsukamoto, and T. Yamamoto. 2007. Molecular cloning, expression and properties of an $\alpha / \beta$-galactoside $\alpha 2,3$-sialyltransferase from Vibrio sp. JT-FAJ-16. J. Biochem. 142:403-412.

13. Tsukamoto, H., Y. Takakura, and T. Yamamoto. 2007. Purification, cloning and expression of an $\alpha / \beta$-galactoside $\alpha$-2,3-sialyltransferase from a luminous marine bacterium, Photobacterium phosphoreum. J. Biol. Chem. 282:29794-29802.

14. Tsukamoto, H., Y. Takakura, T. Mine, and T. Yamamoto. 2008. Photobacterium sp. JT-ISH-224 produces two sialyltransferases. $\alpha$-/ $\beta$-galactoside $\alpha 2,3$-sialyltransferase and $\beta$-galactoside $\alpha 2,6$ sialyltransferase. J. Biochem. 143:187-197.

15. Varki, A. 1993. Biological roles of oligosaccharides: All of the theories are correct. Glycobiology 3:97-130.

16. Vimr, E.R., K.A. Kalivoda, E.L. Denzo, and S.M. Steenbergen. 2004 Diversity of microbial sialic acid metabolism. Microbiol. Mol. Biol. Rev. 68:132-153.

17. Yamamoto, T., M. Nakashizuka, H. Kodama, Y. Kajihara, and I. Terada. 1996. Purification and characterization of a marine bacterial $\beta$-galactoside $\alpha 2,6$-sialyltransferase from Photobacterium damsela JT0160. J. Biochem. 120:104-110.

18. Yamamoto, T., Y. Takakura, and H. Tsukamoto. 2006. Bacterial sialyltransferase. Trends Glycosci. Glycotechnol. 18:253-265.

19. Yamamoto, T., Y. Hamada, M. Ichikawa, H. Kajiwara, T. Mine, H. Tsukamoto, and Y. Takakura. 2007. A $\beta$-galactoside $\alpha 2,6-$ sialyltransferase produced by a marine bacterium, Photobacterium leiognathi JT-SHIZ-145, is active at pH 8. Glycobiology 17:11671174 .

20. Yuki, N., K. Suzuki, M. Koga, et al. 2004. Carbohydrate mimicry between human ganglioside GM1 and Campylobacter jejuni lipooligosaccharide causes Guillain-Barré Syndrome. Proc. Natl. Acad. Sci. USA 101:11404-11409. 